\title{
PULSE: A MAC Protocol for RFID Networks`
}

\author{
Shailesh M. Birari and Sridhar Iyer \\ K.R. School of Information Technology, \\ Indian Institute of Technology, Powai, Mumbai 400 076, India \\ \{shailesh, sri\}@it.iitb.ac.in
}

\begin{abstract}
The reader collision problem occurs when the signal from one reader interferes with the signal from other readers. Solutions like RTS-CTS are not applicable because a reader may communicate with multiple tags simultaneously. In this paper, we describe Pulse, a distributed protocol to reduce reader collisions. The operation of the Pulse protocol is based on periodic beaconing on a separate control channel by the reader, while it is reading the tags. The protocol functions effectively with fixed as well as mobile RFID readers. We show, using simulation in QualNet, that using Pulse protocol, the throughput (overall read rate) is increased by as high as $98 \%$ (with 49 readers) as compared to "Listen Before Talk" (CSMA) and by 337\%(with 9 readers) as compared to Colorwave. We also present an analytical model for our protocol in a single hop scenario.
\end{abstract}

\section{Introduction}

An RFID system consists of an RFID reader and a set of RFID tags. The reader uses radio waves to communicate with the tag. A tag may be active(powered by an external battery) or passive(powered by energy in the reader's signals). Since the signal from a passive tag to the reader is a reflected signal, the read range of a reader is very limited. Not all applications require "always-on" / real-time sensing of the item to be tracked. So a large deployment of fixed readers to cover the area is an overkill. Instead periodic walk-through of fewer mobile readers would suffice to cover the deployment area thus reducing the cost of deployment.

Many applications require readers to operate in close proximity of each other. Due to proximity, the signals from one reader might interfere with the signals from other readers. This interference is called reader collision [1.

Reader to Reader interference arises when stronger signal from a reader interfere with the weak reflected signal from a tag. For example, in fig. 1, $R_{1}$ lies in interference region of reader $R_{2}$. The reflected signals reaching reader $R_{1}$ from $\operatorname{tag} T_{1}$, can easily get distorted by signals from $R_{2}$. Note that such interference is possible even when the read range of the two readers do not overlap.

Multiple reader to tag interference arises when more than one reader try to read the same tag simultaneously. In fig. 2, the read range of the two readers overlap. Hence the signals from $R_{1}$ and $R_{2}$ might interfere at tag $T_{1}$. In such

\footnotetext{
^ This work was partly supported by Persistent Systems Pvt. Ltd.www.persistent.co.in.
} 


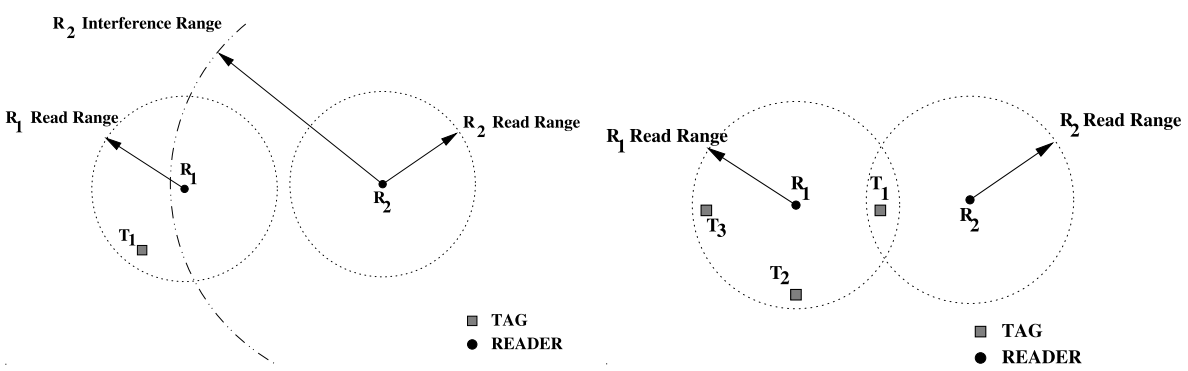

Fig. 1. Reader to Reader Interference

Fig. 2. Reader to Tag Interference

case, $T_{1}$ can not decipher any query and the tag is read neither by $R_{1}$ nor by $R_{2}$. Due to reader collisions, $R_{1}$ will be able to read $T_{2}$ and $T_{3}$ but it may not be able to read the tag $T_{1}$. In such case, $R_{1}$ will indicate presence of 2 tags instead of 3 .

Apart from such incorrect operations, reader collisions also result in reduction of the overall read rate of the RFID system. Hence reducing these reader collisions is essential. Morever this problem is aggravated in case of mobile/handheld readers.

Standard multiple access mechanisms cannot be directly applied to RFID systems due to the following reasons.

- FDMA: With FDMA, the interfering readers use different frequencies to communicate with the tags. Since the RFID tags do not have any frequency selectivity, they cannot select a particular reader frequency for communication. Hence FDMA is not a practical solution in RFID systems.

- TDMA: With TDMA, the interfering readers are alloted different time slots thus avoiding simultaneous transmissions. However this is similar to the well known coloring problem in graph theory[1] which is an NP-hard problem[1]. Also because of mobility, non interfering readers may move closer and start interfering making TDMA an inefficient solution.

- CSMA: In fig. 3, the read ranges of the two readers donot overlap. However, the signals from reader $R_{2}$ can interfere with the signals from reader $R_{1}$ at tag $\mathrm{T}$. This case can also happen when the two readers are not in each other's sensing range making carrier sensing(and hence CSMA) ineffective in RFID networks.

- CDMA: CDMA will require extra circuitry at the tag which will increase the cost of the tags. Also code assignment to all the tags at the deployment site may be a complicated job.

FDMA, TDMA and CSMA are discussed in more detail in section 2 Standard anti-collision protocols like RTS-CTS cannot be directly applied to RFID systems due to following reasons.

- In case of traditional wireless networks, only one node has to send a CTS back to the sender. However in RFID, if a reader broadcasts an RTS, all 


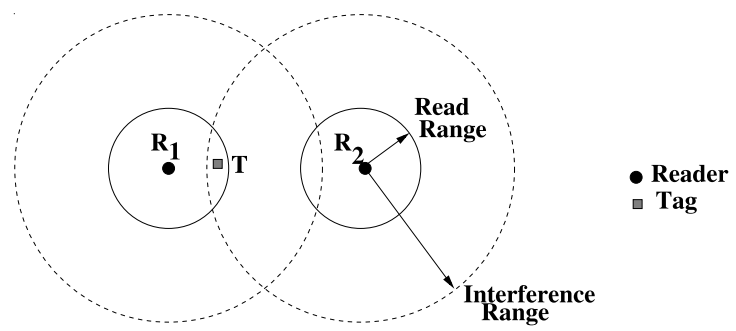

Fig. 3. Reader Collision making carrier sensing ineffective

tags in the read range need to send back a CTS to the reader. This demands another anticollision mechanism for these CTS which will make the protocol more complicated.

- Also there are chances that a tag ( say $T_{1}$ ) may not receive an RTS due to collision while other tag ( say $T_{2}$ ) may receive it. In such case, a CTS from $T_{2}$ is not a guarantee that there is no collision in the read range of the reader.

We propose a distributed protocol, Pulse, based on a beaconing mechanism. While a reader is reading the tags, it periodically broadcasts a beacon on a separate control channel. Any other reader that wants to communicate with the tags, first senses the control channel for a beacon. If it does not receive any beacon for a specified amount of time, it transmits a beacon and starts communicating with the tags. It then continues to periodically transmit a beacon as long as it is communicating with the tags.

\section{Related Work}

The Class 1 Generation 2 UHF standard 2] ratified by EPCGlobal 3 ] uses spectral planning(FDMA). It seperates the reader transmissions and the tag transmissions spectrally such that tags collide with tags but not with readers and readers collide with readers but not with tags. Such seperation solves the reader to reader interference since the reader transmissions and tag transmissions are on seperate frequency channels. However the tags donot have frequency selectivity. Hence when two readers using separate frequency communicate with the tag simultaneously, it will lead to collision at the tags. Thus multiple reader to tag interference still exists in this standard.

Colorwave 4] is a distributed TDMA based algorithm, where each reader chooses a random time slot to transmit. If it collides, it selects a new timeslot and sends a kick to all its neighbours to indicate selection of new timeslot. If any neighbour has the same color, it chooses a new color and sends a kick (small control packet) and this continues. If the percentage of successful transmission goes below certain threshold, the maxColors is incremented and if the percentage increases beyond certain threshold, the maxColors is decremented. More details about the algorithm can be found in [4]. 
Colorwave requires time synchronisation between readers. Also, Colorwave assumes that the readers are able to detect collisions in the RFID system. However it may not be practical for a reader alone to detect the collisions that happen at the tags unless the tags take part in the collision detection.

ETSI EN 302 208 [5] is an evolving standard being developed for RFID readers. It has a CSMA based protocol called "Listen Before Talk". The reader first listens on the data channel for any on-going communication for a specified minimum time. If the channel is idle for that time, it starts reading the tags. If the channel is not idle, it chooses a random backoff. However as described earlier, the readers may not be able to detect collision by carrier sensing alone.

\section{Pulse Protocol}

RFID networks also suffer from the hidden terminal problem. As seen in figure 3. $R_{1}$ and $R_{2}$ are not in each other's sensing region, but signals from $R_{2}$ might interfere with signals from $R_{1}$ at tag $T$. For such a scenario, a notification mechanism is required between $R_{1}$ and $R_{2}$ such that $R_{2}$ is informed of $R_{1}$ 's transmissions before it communicates with the tag. We propose to have this notification through a broadcast message called "beacon" on a seperate control channel.

The communication range in the control channel is such that, any two readers that can interfere with each other on the data channel (channel used to read the tags), are able to communicate on the control channel. Thus in fig 3 since $R_{1}$ and $R_{2}$ interfere with each other on the data channel, they will be able to communicate on the control channel. This can be achieved by making the readers transmit at a higher power on the control channel than the data channel. The control channel can simply be a sub-band in the RFID spectrum apart from those used for reader-tag communication. Hence transmission on the control channel will not affect any on-going communication on the data channel.

\subsection{Description}

Pulse protocol is present only at the reader since the tags do not take part in the collision avoidance. The data channel is used for reader-tag communication whereas the control channel is used for reader-reader communication. We assume that the reader is able to simultaneously receive on both the control and the data channel.

Following is an overview of the Pulse protocol.

- Before communicating with the tags, a reader has to wait in the state WAIT$I N G$ for a minimum time $T_{\min }$ which is thrice the beacon interval. The time $T_{\min }$ is analogous to the DIFS time in 802.11 protocol[7]. Everytime it receives a beacon in this state, it resets its waiting time to $T_{m i n}$.

- After $T_{\min }$ time has elapsed and it did not receive any beacon, the reader concludes that there is no other reader in the neighbourhood which is reading the tags. Hence it enters a contention phase and chooses a random backoff 


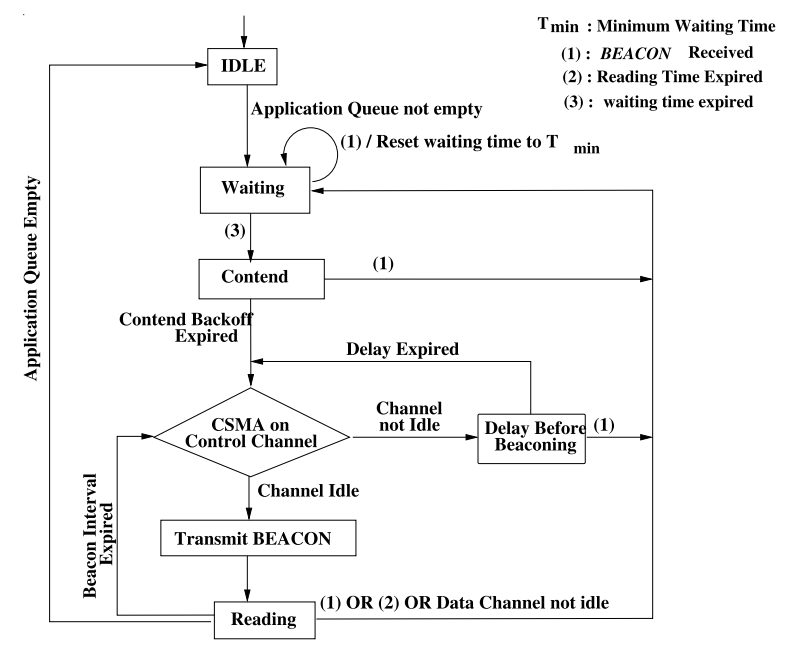

Fig. 4. Flow Chart for Pulse

time (contend_backoff) from the interval $[0 \ldots C W]$. If it chooses $i$, it waits for $i$ beacon intervals in state CONTEND. If it now receives a beacon, it has lost this cycle and waits for the next cycle, i.e until it does not receive a beacon for atleast $T_{\min }$ time. If the randomized backoff time is over and the reader did not receive any beacon, the reader sends a beacon on the control channel and starts communicating with the tags on the data channel. This randomized backoff helps to avoid collisions between readers. contend_backoff is a multiple of beacon intervals to improve fairness.

- While the reader is communicating with the tags, the reader sends a beacon on the control channel every beacon interval. This beacon acts as a notification to the neighbouring readers so that they can withhold their communication with the tags and thus avoid possible collisions. After the communication with the tags is over, the reader again waits in the WAITING state and the cycle continues.

- Everytime the reader sends a beacon, it first senses the control channel. If the control channel is busy, it continues to sense the control channel. As soon as the channel gets idle, the reader waits for a random delay ( $d e$ lay_before_beaconing) and senses the channel again to send the beacon. This random delay is a multiple of the beacon propagation delay and helps to avoid collisions - otherwise many readers would simultaneously send the beacon after the channel became idle.

Fig. 4 shows the detailed flowchart and fig. 5 shows the detailed algorithm for the Pulse protocol.

The contend_backoff and the delay_before_beaconing in the protocol are similar to the backoffs in general wireless networks, they are decreased as long as the control channel is sensed idle, stopped when a transmission is detected, and 


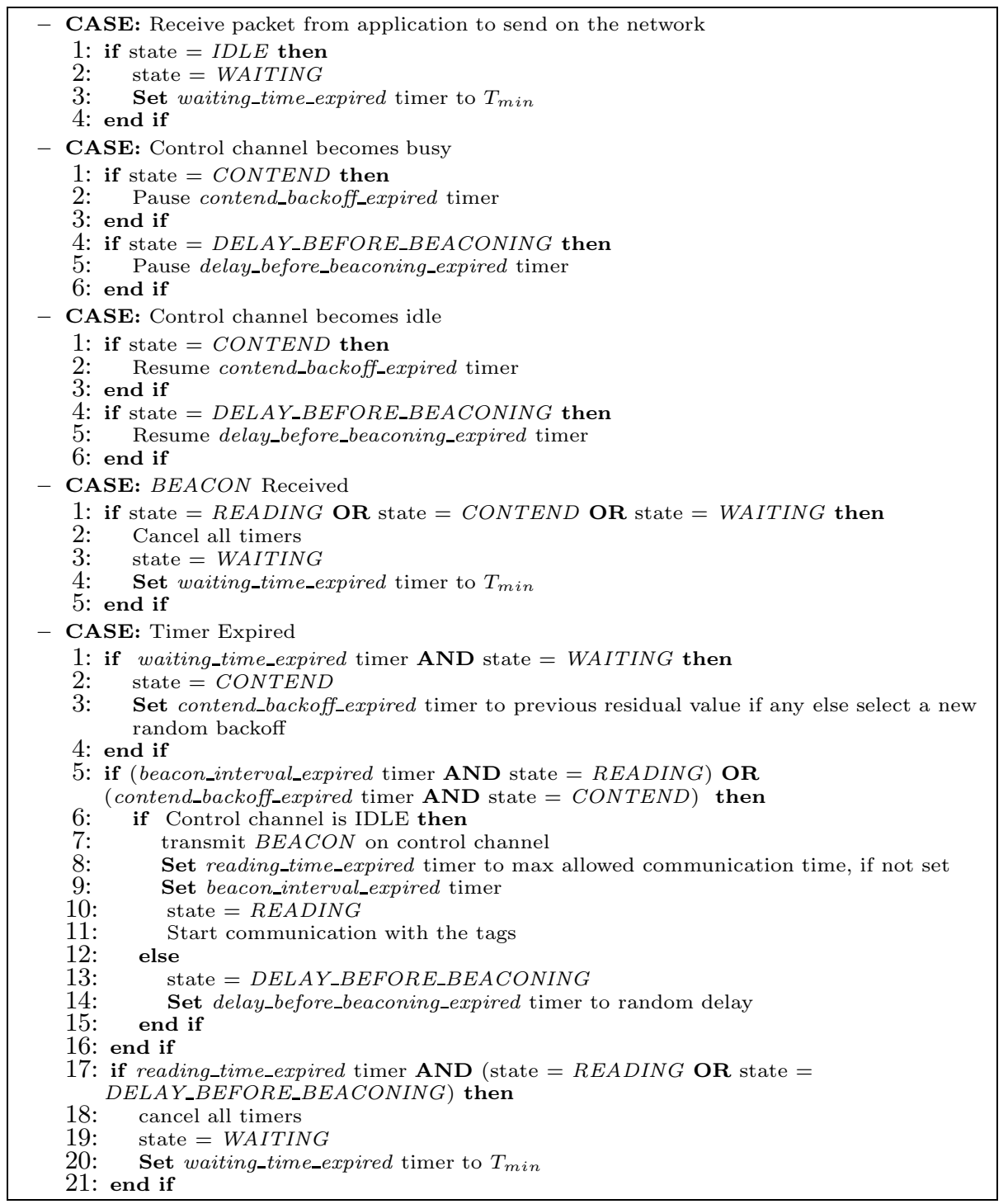

Fig. 5. Pulse Protocol Algorithm

reactivated when the control channel is sensed idle again. Also, if the reader receives a beacon during backoff (contend_backoff), in the contention phase, it stores the residual backoff timer and then waits for the next chance, i.e until it does not receive a beacon for atleast $T_{\min }$ time. It then uses this residual backoff time. This is done only to improve fairness amongst readers. Although the protocol seems to be simple, we show using simulations that it is effective in both static and mobile networks. 


\section{Simulation Experiments}

\subsection{Simulation Model}

We have simulated the UHF RFID network in QualNet simulator [8] with data channel frequency as $915 \mathrm{MHz}$ and the control channel frequency as $930 \mathrm{MHz}$. We assumed: No inter channel interference between the data and the control channel, Free space propagation path loss, no fading, SNR based signal reception(SNR = 10), omni-directional antennas, $2 \mathrm{Mbps}$ data rate, $-91 \mathrm{dBm}$ Radio Rx sensitivity and $-81 \mathrm{dBm} \mathrm{Rx}$ threshold, negligible data processing delay and channel switching delay and packet collision as the only cause of packet loss. We also adjusted the transmission power of the RFID node $-45 \mathrm{dBm}$, to make the read range $\sim 5$ feet as is the case with UHF RFID readers.

With these parameters the read range, sensing range and the interference range are 5.31 feet(1.62 meters), 17.71 feet( 5.4 meters) and 23.29 feet(7.1 meters) respectively. Here the interference range is the maximum distance upto which a reader's transmission can interfere with another reader-tag communication. Thus the beacon range should be atleast equal to the interference range inorder to make this protocol effective.

We define the Beacon Range Factor(BRF) as the ratio of the control channel transmission power to the data channel transmission power. According to 9], the power received at a receiver is inversely proportional to the square of the distance between the transmitter and receiver. Thus, BRF is given by 10 ]

$$
B R F=\frac{P_{\text {Beacon }}}{P_{\text {Data }}}=\frac{r_{\text {Beacon }}^{2}}{r_{\text {Data }}^{2}}
$$

Thus with data range as 1.62 meters, inorder to have a beacon range of 7.1 meters, we require a BRF of 19.2.

\subsection{Performance Metrics}

A query is said to be successfully sent if it is sent by a reader and is successfully received by all the tags in the read range i.e. it does not collide with any other query in the network. We define the system throughput and the percentage efficiency as follows.

$$
\begin{gathered}
\text { System Throughput }=\frac{\text { Total queries sent successfully(by all readers })}{\text { Total time }} \\
\text { System Efficiency }(\%)=\frac{\text { Total queries sent successfully }(\text { by all readers }) \times 100}{\text { Total queries sent(successful }+ \text { collided }) \text { by all readers }}
\end{gathered}
$$

In general, the tag identification is through a query-response protocol where the reader sends a query and the tag responds with its unique identification number. Higher the number of queries sent successfully, higher the throughput, and hence higher would be the number of tags identified by the readers. Percentage efficiency reflects the ability of a protocol to detect a possibility of collision at the tags and hence avoid unnecessary transmissions. An improvement in throughput 
indicates an improvement in the read rate whereas an improvement in the efficiency indicates reduction in collisions. Thus throughput and efficiency together define the effectiveness of the protocol. Through simulations we show that Pulse protocol is effective in both the dimensions.

\subsection{Simulation Scenarios}

We used the following simulation setup for running the experiments.

- Tag setup: We used a field of 10 meter X 10 meter area, with 400 tags forming a grid of $20 \mathrm{X} 20$. The tags were placed throughout the simulation field with 0.5 meter interval so that most of the collisions in the field would be detected by these tags.

- Fixed Readers: For fixed reader simulation, all the readers were randomly placed in the field. We used 20 random topologies with 3 different seeds in each case giving a total of 60 simulations per protocol.

- Mobile Readers: For simulation of mobile readers, the initial placement of readers was a uniform grid of readers. We used a random way point mobility with low speed of 0.5 to 2 meters per second and 10 random seeds.

For simulation, the RFID application generated a packet(query) to be sent to the tags with exponential interarrival time of average $500 \mu \mathrm{sec}$ throughout the simulation time of 60 seconds.

\subsection{Compared Protocols}

We compared our Pulse protocol with Aloha protocol, CSMA protocol [5] [6] and Colorwave. A reader with Aloha protocol assumes that it is the only reader communicating with the tag. Hence when the reader wants to communicate with the tags, it simply starts its transmission without applying any collision avoidance. The CSMA protocol is similar to ETSI EN 302 208[5] 6] with a listen time of $15 \mathrm{msec}$. For Colorwave protocol, we used the time slot of $10 \mathrm{msec}$. Rest of the experiment setup for Colorwave was as given in [4]

We set the beacon interval of Pulse protocol as $5 \mathrm{msec}$ and $T_{\min }$ same as the listen time in CSMA i.e 15msec. Using similar settings for both the protocols help us evaluate the MAC protocols in an unbiased manner.

\section{Results}

Keeping $\mathrm{BRF}=28$ and beacon interval $=5 \mathrm{msec}$, we did the comparison initially on a 25 reader topology followed by topologies with different number of readers (4...64). We also studied the effect of BRF and beaconing interval on throughput and efficiency of Pulse.

\subsection{Throughput}

25 Reader Topology: Fig. [6] shows system throughput with different protocols. 


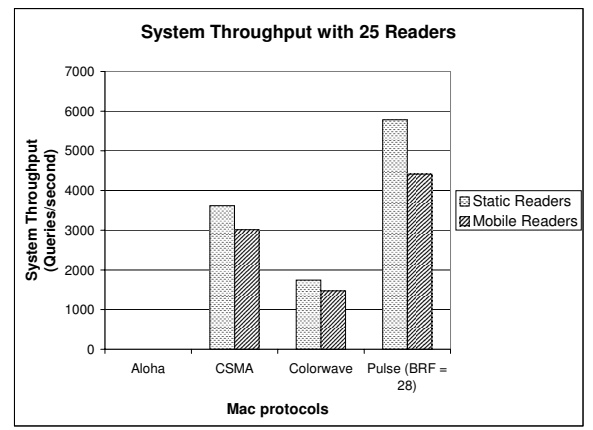

Fig. 6. Throughput comparison with 25 readers

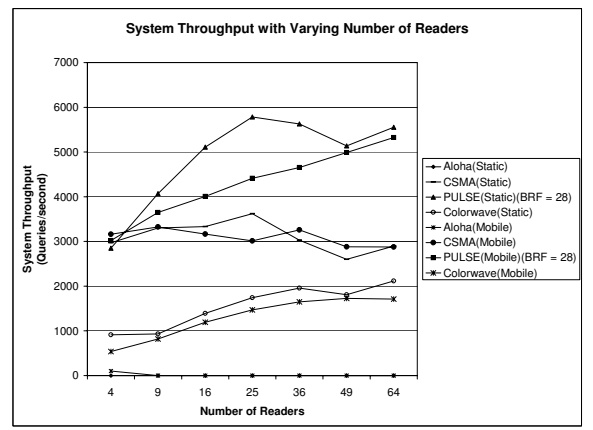

Fig. 7. Throughput comparison with different number of readers

- With Aloha protocol, almost every transmission in the system collided because readers with aloha protocol do not apply any collision avoidance.

- CSMA has better throughput than Aloha because carrier sensing is succesful in avoiding collision with readers within the sensing range. However number of collisions using CSMA is still high due to the hidden terminal problem.

- In colorwave, because of the distributed timeslot mechanism, the timeslots are underutilised thus showing lower throughput.

- In Pulse, these collisions are avoided because the beacon sent by a reader acts as a notification to the neighbouring readers(including hidden nodes), which then withhold their transmission thus avoiding collisions. Pulse shows throughput improvement of $60 \%$ as compared to CSMA and $232 \%$ as compared to Colorwave in static topology.

- Even in case of mobility, Pulse remains to be effective with throughput improvement of about $46 \%$ as compared to CSMA and $200 \%$ as compared to Colorwave.

Varying Number of Readers: Fig. 7 shows the graph of throughput with varying number of readers in the system. Following are the observations:

- Aloha continues to show negligible throughput.

- As the number of readers in the system are increased, the throughput of CSMA protocol does not increase. Hence unable to cater to dense networks.

- Pulse protocol shows better throughput in all topologies as compared to both colorwave and CSMA protocol. It shows an improvement of as high as $98 \%$ (with 49 readers) over CSMA and 337\% (with 9 readers) over Colorwave.

- Using Pulse protocol, the throughput of the system keeps on increasing as the number of readers in the system is increased upto a saturation point after which the throughput stops increasing even if the number of readers is increased. For example for $\mathrm{BRF}=28,25$ readers is the saturation point. Hence if the throughput of the system is of prime importance, no more than the saturation number of readers should be deployed.

- Note that Pulse is effective even in a highly dense network of 64 readers. 


\subsection{Efficiency}

25 Reader Topology: Fig.8 shows system efficiency with different MAC protocols.

- the efficiency with CSMA barely crosses $50 \%$ which means that $50 \%$ of the transmissions in the network are wasted due to collision.

- Using Colorwave, the efficiency is almost $100 \%$ however, colorwave fails to give better throughput than Pulse.

- With Pulse, the efficiency is above $99 \%$ with both static and mobile reader network. Thus Pulse is successful in detecting possibility of collisions and thus avoid the same.

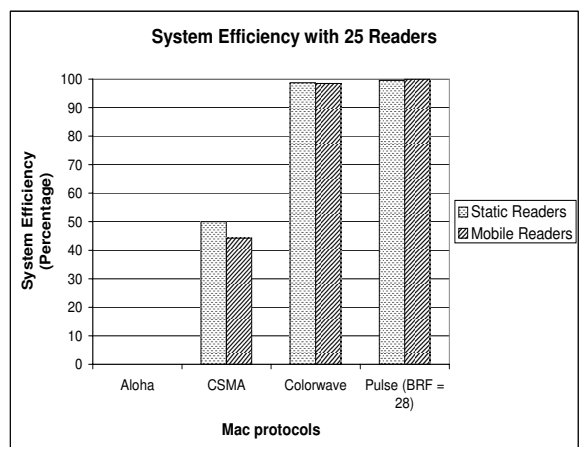

Fig. 8. Efficiency with 25 Readers

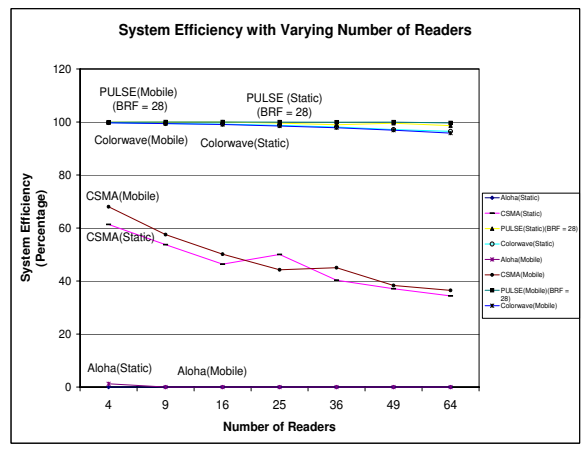

Fig. 9. Efficiency with Varying Number of Readers

Varying Number of Readers: Fig. 9 shows the graph for the same.

- With Aloha protocol, the efficiency is negligible in all the experiments.

- As seen, the efficiency of CSMA keeps on decreasing as the number of readers go on increasing. As the density of the network increases, the number of hidden terminals increase thus reducing the efficiency.

- Pulse protocol overcomes the hidden terminal problem through a beacon and hence the efficiency of the system is above $95 \%$ in all topologies.

Thus Pulse is definitely an improvment over the existing solutions in both the dimensions of throughput and efficiency. It remains to be effective even in a highly dense mobile network.

We further tested the effect of the protocol parameters, BRF and beaconing interval, on the system throughput and efficiency. We found that BRF of 28 gives the highest throughput. We also found that change in beacon interval does not show any significant change in the system throughput. We also theoretically analysed Pulse for which we assumed that all the readers in the network are in each others' communication range on the control channel. We divided the time into cycles and calculated the average number of queries transmitted per cycle which gives the overall system throughput. More details can be found in [10. 


\section{Conclusion and Future Work}

The reader collision problem in RFID networks is a hindrance for the proliferation of RFID. We presented a distributed protocol, Pulse, for an RFID network which uses a beaconing mechanism by sending periodic beacon on the control channel. Although the protocol is simple, we have shown that it mitigates the reader collision problem. It reduces the reader collisions to $1-2 \%$ and also increases the read rate of the system by as high as $98 \%$ as compared to CSMA. It requires very less overhead on the reader side and absolutely no support on the tag side. Our protocol is also very effective in a mobile scenario facilitating the use of mobile readers which is a cost effective solution for many applications.

We did not account for any channel switching delay in our simulations. However we believe it to be negligible as compared to the beacon interval. Ofcourse, the Pulse protocol demands for some extra circuitry on the receiver end of a reader. However Pulse protocol increases the throughput considerably. It also promotes the use of lesser number of readers by being effective in a mobile scenario. We believe this performance gain and reduction in number of readers required is high enough to offset the hardware modification required by this protocol.

Further research can involve porting of the Pulse protocol to readers with multiple data channels. Further analysis will lead to insights on the ideal parameters like the beaconing interval, waiting time and the maximum capacity of the protocol.

\section{References}

1. Daniel W. Engels. The reader Collision Problem. Technical report, epcglobal.org, 2002.

2. Chris Diorio. Class 1 generation 2, uhf rfid. CTAN: www.autoid.org/SC31/2004/ dec/SG3_200411_430_Gen2Update.pdf, December 2004.

3. Electronic Product Code. http://www.epcglobalinc.org.

4. J. Waldrop, D. W. Engels, and S. E. Sarma. Colorwave: An Anticollison Algorithm for the Reader Collision Problem. In IEEE Wireless Communications and Networking Conference (WCNC), 2003.

5. ETSI EN 302 208-1 v1.1.1, September 2004. CTAN: http://www.etsi.org

6. ETSI EN 302 208-2 v1.1.1, September 2004. CTAN: http://www.etsi.org

7. IEEE Computer Society LAN MAN Standards Committee. Wireless LAN Medium Access Control (MAC) and Physical Layer(PHY) Specifications. In ANSI/IEEE Std. 802.11, 1999 Edition, The Institute of Electrical and Electronics Engineers. New York, 1999.

8. Qualnet Simulator 3.6. http://www.qualnet.com

9. Prashant Krishnamurthy Kaveh Pahlavan. Principles of Wireless Networks. Pearson Education, 2002.

10. Shailesh Birari. Mitigating the Reader Collision Problem in RFID Networks in Mobile Readers. Master's thesis, Indian Institure of Technology, Bombay, July 2005 . 